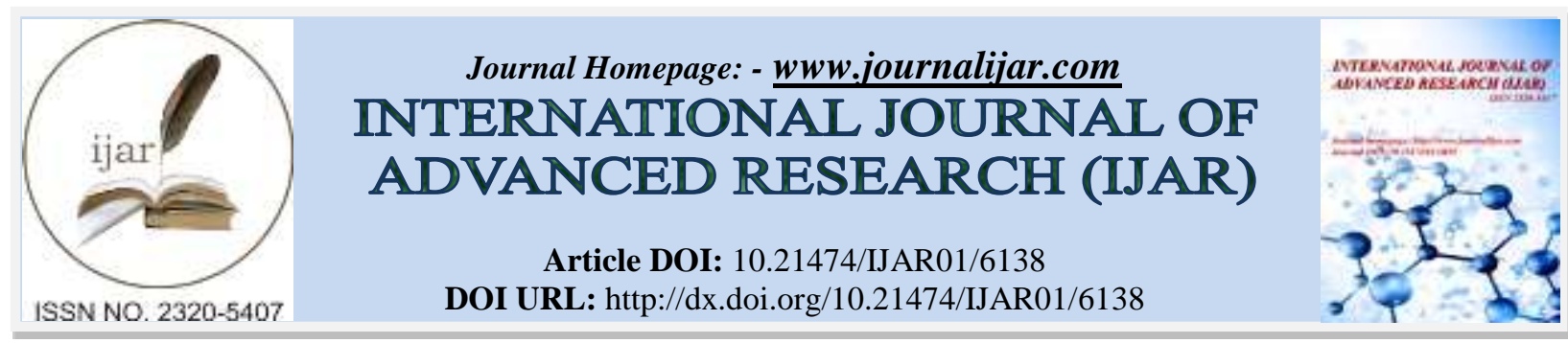

RESEARCH ARTICLE

\title{
CONSERVATIVE TREATMENT OF BREAST CANCER, EXPERIENCE OF THE GENERAL SURGERY DEPARTMENT OF THE AVICENNE MILITARY HOSPITAL.
}

\author{
Mohammed Lahkim, Mohammed Es-said Ramraoui, Mohammed Jaouad Fassi Fihri, Ahmed Elguezzar, \\ Ahmed Elkhader, Rachid El Barni and Abdessamad Achour. \\ Service de chirurgie générale, hopital militaire Avicenne, 40000, Marrakech, Maroc.
}

\section{Manuscript Info}

Manuscript History

Received: 22 October 2017

Final Accepted: 24 November 2017

Published: December 2017

Key words:-

Breast cancer, Breast-conserving treatment, Limits of excision, Local recidivism.

\begin{abstract}
Breast cancer is currently the most common cancer in women, and is a major diagnostic and therapeutic problem. The radio-surgical conservatrice therapeutic management has become a standard for most tumors : stages I and II. Furthermore, the use of preoperative treatment extends the indications of conservative treatment which was initiall limited to tumors less than $3 \mathrm{~cm}$, unifocal, and non-inflammatory to larger tumors.

Our study reports 20 patients cases of breast cancer, collected at the surgical department's of the military hospital avicenne marrakech's, over a period of 2 years from junuary 2015 to december 2016 who underwent conservative tratment.
\end{abstract}

Copy Right, IJAR, 2017,. All rights reserved.

\section{Introduction:-}

Le cancer du sein est le cancer le plus fréquent chez la femme dans le monde et pose un véritable problème diagnostique et thérapeutique. Le traitement conservateur radio-chirurgical est devenu un standard pour la plupart des tumeurs stade I et II du cancer du sein [1].

Les buts de ce travail sont d'évaluer les indications d'une chirurgie conservatrice pour un cancer du sein et décrire les modalités techniques, d'étudier l'impact de ce traitement sur la survie sans récidive et sans métastase et déterminer les facteurs pronostiques et d'évaluer les résultats anatomiques et esthétiques.

\section{Matériels Et Méthodes:-}

Il s'agit d'une étude rétrospective mené au service de chirurgie générale de l'hôpital militaire Avicenne sur une période de deux ans entre 2015 et 2016, durant laquelle 60 cas de cancer du sein chez la femme ont été pris en charge et dont 20 cas ont bénéficié d'un traitement conservateur.

\section{Résultats:-}

Durant la période de notre étude 60 cas de cancer du sein ont été pris en charge dans notre service dont 20 patientes ont bénéficié d'une chirurgie conservatrice, soit une fréquence de $33,33 \%$.

L'âge moyen des patientes était de 53 ans avec des extrêmes allant de 24 à 82 ans et $40 \%$ de patientes âgées de moins de 45 ans. 
L'âge moyen des premières règles était de 14,5 ans. Parmi les 20 patientes, 15 étaient ménopausées (soit $75 \%$ ) dont aucune n'a reçu un traitement hormonal substitutif et 5 étaient encore en activité génitale (soit $25 \%$ ).

$55 \%$ de nos patientes prenaient une contraception hormonale de type oestroprogestatif.

$74 \%$ de nos patientes étaient multipares dont $44 \%$ avaient plus $\mathrm{d}$ trois enfants. L'âge de la première grossesse était inférieur à 30 ans chez $90 \%$ des patientes.

L'allaitement a été mentionné chez 75\% des patientes cependant la durée n'a pas été précisé dans notre étude.

Aucun antécédent personnel ou familial de cancer du sein n'a été noté chez nos patientes. Par contre on note deux cas de tumeur bénigne et un cas de cancer de l'ovaire.

Le délai moyen entre l'apparition des symptômes est la première consultation varie entre 1 et 5 mois avec une moyenne de 2,75 mois. La découverte d'un nodule du sein était le motif de consultation le plus fréquent avec 14 cas (soit 70\%) suivi de la mastodynie dans 3 cas, un cas d'écoulement mamelonaire et un cas de découverte fortuite lors d'une mammographie de dépistage.

Le sein droit était le plus atteint avec une fréquence de 55\%, le sein gauche chez $40 \%$ des patientes et un seul cas de localisation bilatérale. Le quadrant supéro-externe étain le plus atteint avec une fréquence de $40 \%$. La taille moyenne des nodules était de $2,68 \mathrm{~cm}$ avec des extrêmes allant de 2 à $4 \mathrm{~cm}$.

La répartition de la taille tumorale clinique selon la classification TNM de l'UICC est représentée dans le tableau suivant:

\begin{tabular}{|l|l|l|}
\hline Taille tumorale & Effectifs & Pourcentage \\
\hline T0 & 0 & $0 \%$ \\
\hline T1 & 6 & $30 \%$ \\
\hline T2 & 10 & $50 \%$ \\
\hline T3 & 1 & $20 \%$ \\
\hline Non précis & 3 & $15 \%$ \\
\hline total & 20 & $100 \%$ \\
\hline
\end{tabular}

Les tumeurs diagnostiquées étaient fixées chez $70 \%$ par rapport au plan cutané. L'examen des aires ganglionnaires a objectivé la présence d'adénopathies axillaires homolatérales chez 6 patientes soit $30 \%$ des cas.

Après classification TNM, on note une prédominance des tumeurs classées T2 retrouvées chez $50 \%$ des patientes et des tumeurs $\mathrm{T} 3$ chez $20 \%$ des cas.

La mammographie était réalisée chez 16 cas soit $80 \%$ et a montré des lésions suspectes de malignité chez 14 patientes et deux cas d'image d'allure bénigne.

L'échographie mammaire a été réalisée chez 15 patiente soit $75 \%$ avec des critères de malignité chez 10 patientes et des adénopathies axillaires chez 6 cas.

Le couple mammographie-échographie réalisé chez 11 cas a permis d'établir une classification ACR de toutes les tumeurs. Ainsi, 63\% ont été classés ACR 5, 27\% classés ACR et 9\% classés ACR 3.

Le diagnostic histologique a été réalisé en préopératoire chez 16 patientes. Ainsi, $70 \%$ des patientes ont bénéficié d'une biopsie et qui a confirmé la présence de cellules malignes de type carcinome infiltrant chez toutes ces patientes. Deux autres patientes ont bénéficié d'une cytoponction confirmant le caractère malin dans un seul cas.

L'examen extemporané a été réalisé chez 4 patientes soit $20 \%$.

Le bilan d'extension n'a objectivé aucune métastase au moment du diagnostic. 
Les récepteurs hormonaux étaient positifs dans $75 \%$ des cas et le statut HER quant à lui variait entre le score 0 et le score 2 chez 8 de nos patientes.

Le dosage du marqueur biologique CA15-3 a été demandé pour toutes les patientes permettant ainsi une surveillance postopératoire.

Les patientes ayant bénéficié d'un traitement conservateur sont au nombre de 20 soit $100 \%$ des cas. Les volumes de résections homolatéraux à la tumeur étaient de $20 \mathrm{~g}$ en moyenne. Le curage axillaire a été effectué chez toutes les patientes. Dans $68 \%$ des cas par la réalisation d'une incision directe du creux axillaire et dans $32 \%$ des cas par l'incision de plastie mammaire.

L'étude de nos marges de résection met en évidence des marges saines dans $65 \%$ des cas. Il existait une atteinte des berges dans 35\% des cas, soit 7 patientes et qui ont été reprises par une mastectomie et une par tumorectomie.

Les tumeurs classées grade I SBR était de 5\%, grade II de l'ordre de $45 \%$ et grade III $30 \%$.

La durée moyenne d'hospitalisation était de 3 jours. Les complications précoces étaient un hématome chez $30 \%$ des patientes, une lymphocèle chez $45 \%$ des patientes, une surinfection chez $10 \%$ des patientes et des troubles sensitifs dans $20 \%$ des cas.

La complication tardive la plus fréquente était le lymphodème dans $20 \%$ des cas.

Toutes nos patientes ont bénéficié d'une chimiothérapie et d'une radiothérapie adjuvantes accompagnant la chirurgie. Quant à l'hormonothérapie, elle a été prescrite dans $75 \%$ des cas.

Nous avons relevé 5 cas présentant des malformations modérées, et 3 présentant une asymétrie mammaire. Pour les autres patientes, le recul n'est pas suffisant pour juger les séquelles.

\section{Discussion:-}

Dans le monde, la fréquence du cancer du sein est variable selon les pays, les taux les plus bas sont ceux des pays asiatiques et africains (l'indice au japon ; 10 cas pour 100000 habitants) et les plus élevés sont les pays occidentaux [1].

Au Maroc, à partir de l'an 2000, le cancer du sein est devenu le premier cancer chez la femme avec une incidence de 27,69/100000 femmes qui est relativement proche de celle des pays du Maghreb [2]. Selon les statistiques de l'institut national d'oncologie de Rabat, le cancer du sein représente $55 \%$ des cancers gynécologiques et $17 \%$ de tous les cancers [3].

Le traitement conservateur du cancer du sein associe une chirurgie mammaire conservatrice consistant en une exérèse tumorale large emportant la tumeur ainsi qu'une marge de tissu mammaire avoisinant macroscopiquement saine à une chirurgie des ganglions axillaires et une irradiation du sein conservé. Ce traitement conservateur est actuellement le traitement de référence des cancers du sein T1 et T2 de petite taille, non inflammatoires. Ce traitement répond à trois objectifs : une survie identique à celle obtenue par la mastectomie, un risque de récidive locale faible et un résultat esthétique satisfaisant [4].

La chirurgie oncoplastique se définit quant à elle, comme l'utilisation de techniques de chirurgie plastique lors du traitement conservateur du cancer du sein. L'exérèse tumorale est corrélée à un geste plastique de comblement du défect glandulaire pour préserver la morphologie du sein et améliorer les résultats esthétiques [4].

Classiquement, le traitement conservateur est proposé pour des tumeurs dont le diamètre est inférieur à $3 \mathrm{~cm}$. Cependant, deux études randomisées $[5,6]$ portant sur des lésions allant jusqu'à $5 \mathrm{~cm}$ ont montré que les taux de survie étaient identiques après traitement conservateur et mastectomie. La difficulté est d'ordre technique : il est plus souvent impossible d'obtenir un résultat esthétique satisfaisant après tumorectomie large pour des lésions de cette taille, sauf en cas de sein très volumineux. En effet, une tumorectomie de très gros volume entraine un risque élevé de déformation mammaire [7]. Dès les années 1987, certaines équipes européennes ont proposé de réaliser un traitement préopératoire afin de réduire le volume tumoral, pour ensuite faire bénéficier la patiente d'un traitement 
conservateur. La chimiothérapie néoadjuvante est actuellement le plus souvent proposée pour un traitement à visée de réduction du volume tumoral mais la radiothérapie et l'hormonothérapie préopératoire sont également possibles. Cependant, tout traitement préopératoire nécessite un examen clinique précis (dimensions tumorales et distance tumeur-mamelon), un bilan radiologique mammographie-échographie est également nécessaire afin de préciser qu'il s'agit bien d'une tumeur unifocale et de pouvoir ultérieurement évaluer la réponse au traitement. Certaines caractéristiques histologiques et biologiques de la tumeur doivent être connues avant de débuter le traitement, car elles pourront être modifiées par celui-ci : la microbiopsie ou un drill biopsie sont systématiques [7].

La radiothérapie préopératoire a été le premier traitement à visée cytoréductrice proposé en cas de cancer du sein volumineux. Une étude de V.Doridot [8] a démontré qu'en cas de réponse tumorale suffisante, cette irradiation préopératoire permet un traitement conservateur du sein, en effectuant une tumorectomie large du reliquat à l'issue de l'irradiation.

A l'institut Curie, une irradiation préopératoire est proposée aux patientes de plus de 65 ans atteintes d'une tumeur unifocale avec des récepteurs hormonaux $\mathrm{RH}+$ de 3 à $7 \mathrm{~cm}$ sans microcalcifications étendues. Une évaluation clinique et radiologique est ensuite effectuée 15 jours après la fin de l'irradiation afin de déterminer la suite du traitement local. Une réponse majeure est définie par une régression tumorale de plus de $50 \%$; ce qui concernait 85\% des patientes de cette série [9]. Dans $77 \%$ des cas, un traitement chirurgical conservateur a pu être proposé six semaines après l'irradiation.

Plusieurs essais ont montré que les taux de conservation mammaire pouvaient être augmentés grâce à une chimiothérapie néoadjuvante. L'analyse de la littérature montre des chiffres de conservation mammaire après chimiothérapie néoadjuvante proche de $50 \%[9,10]$. Cependant, cette chimiothérapie première n'apporte pas de bénéfice de survie par rapport à une chimiothérapie postopératoire $[9,10]$. La chimiothérapie comprend 4 à 6 cycles selon les protocoles. L'intervention est programmée un mois après la dernière cure. En cas de réponse tumorale partielle ou complète, autorisant un traitement conservateur, une tumorectomie et un curage axillaire sont alors pratiqués. Dans le cas contraire, une mastectomie reste le traitement standard, même s'il est parfois possible de délivrer une radiothérapie préopératoire de rattrapage, un mois après la dernière cure. Le taux de réponse clinique est alors de 60\%, permettant encore le plus souvent un traitement conservateur [11].

Le tamoxifène est la molécule la plus étudiée dans l'hormonothérapie. Une analyse rétrospective chez 199 patientes âgées de 50 à 70 ans qui ont reçu une hormonothérapie néoadjuvante a montré un taux de conservation mammaire de $54 \%$ pour les femmes ayant une tumeur opérable d'emblée et de $44 \%$ pour celle ayant une tumeur localement évoluée [12]. Dans notre série aucun traitement néoadjuvant n'a été prescrit.

Un risque de récidive existe après traitement conservateur. Mais sa survenue n'a pas d'influence sur le taux de survie à 10 ans. La récidive locale constitue un échec du traitement et impose une nouvelle chirurgie avec souvent une mastectomie dans la mesure où le remodelage glandulaire de la plastie mammaire fausse les repères de la tumeur initiale. Ce risque de récidive locale persiste tout au long de la vie de la patiente. Dans tous les essais randomisés, le taux de récidive locale après traitement conservateur est compris entre 4 et $8 \%$ à cinq ans $[13,14,15]$. Trois facteurs de risque de récidive ont fait l'objet d'un consensus :

1. L'âge : le risque de récidive mammaire diminue avec l'âge [13]

2. Les emboles tumoraux : leur présence est un facteur de récidive locale. Il est de $25 \%$ à 10 ans contre $8 \%$ lorsqu'ils n'existent pas $[13,15,16]$

3. L'atteinte des limites d'exérèse augmente le taux de risque de récidive locale. Ce taux est de $28 \%$ à dix ans si l'exérèse est incomplète [17].

Dans 20 à 30\% des cas, le TC s'accompagne de séquelles liées à la chirurgie et à l'irradiation [18] . Les séquelles consistent essentiellement en une asymétrie de volume ou une asymétrie de position de l'aréole (41.9\% des cas dans notre série); une déformation du sein par perte de substance glandulaire ou rétraction cutanée ( $9.4 \%$ de nos cas); une cicatrice élargie, rétractile et inesthétique ou encore une bride cicatricielle. L'évaluation des résultats esthétiques peut être standardisée par l'utilisation de classifications des séquelles esthétiques du TC. [18] . Ces classifications sont proposées comme guide pour aider les chirurgiens à planifier la reconstruction du sein. 


\section{Conclusion:-}

Le choix de la chirurgie conservatrice, lorsque les indications sont bien posées, constitue un appoint supplémentaire dans les méthodes chirurgicales de traitement du cancer du sein. Cependant ces indications doivent être réfléchies et le résultat carcinologique doit être mis en avant, afin d'obtenir des limites de résection saines et pour garantir une meilleure survie sans récidive.

\section{Conflits d'intérêts:-}

Les auteurs ne déclarent aucun conflit d'intérêts.

\section{Références:-}

1. Ferlay J et al. (2007) Estimates of the cancer incidence and mortalityin europe in $2006 \mathrm{HC}$,InVS,INCa, et al (2010)

2. Projections de l'incidence et de la mortalité par cancer en France en 2010. Rapport technique. Avril 2010 P.Kamina ; Atlas d'antomie humaine. P24 VAN DONGEN JA, BARTELINK H et al

3. Factors influencing local relapse and survival and results of salvage treatment after breast-conserving therapy in operable breat cancer : EORTC trial 10801, breast conservation compared with mastectomy in TNM stage I and II breast cancer. Eur J cancer $1992 ; 28: 801-111$

4. Jacobson JA, Danforth DN, Cowan KH, Steinberg SM, Pierce L et al

5. Ten years results of a comparaison of conservation with mastectomy in the treatment of stage I and II breast cancer. N Engl J Med $1995 ; 332: 907-11$

6. Veronesi U, Volerrani F, Luini A, Saccozzi R et al

7. Quandrectomy versus lumpectomy for small size breast cancer. Eur J cancer $1990 ; 26(6): 671-3$

8. V.Doridot et al / cancer / Radiotherapie 8 (2004) 21-28 (Elseiver article) Schwartzberg BS, Goates JJ, Keeler SA, Moore JA

9. Use of advanced breast biopsies : review of 150 consecutive biopsies. J Am Coll Surg $2000 ; 191: 9-15$

10. Mauriac L, Durand M, Floquet A, Debled M et al

11. Neoadjuvant chemotherapy for operable breast carcinoma larger than $3 \mathrm{~cm}$ a unicenter randomized trial with 124 month follow-up. Ann oncol $1999 ; 10: 47-52$ Insitut Bergonie Bordeaux Groupe Sein (IBBGS) Scholl SM, Pierga JY, Vilcoq JR, Durand JC et al

12. Neoadjuvant vs adjuvant chemotherapy in premenopausal patients with tumours considered too large for breast conserving surgery ; preliminary results of a randomised trial :S6. Eur J cancer $1994 ; 30$ :645-52 Mauriac L, Durand $\mathrm{M}$ et al

13. First line tamoxifene for invasive hormonal sensitive non metastatic breast carcinomas in young postmenopausal patients. Breast cancer Res Treat $1999 ; 57: 435$. Gage I, Schnitt SJ. Troyan SL et al

14. Pathologic margin involvement and the risk of recurrence in patients treated with breast-conserving therapy cancer $1996 ; 78: 1921-8$ Fowble B, Solin IJ et al

15. Breast recurrence following conservative surgery and radiation : patients of failure prognosis and radiation : patterns of failure, prognosis and pathologic finding from mastectomy specimens with implicatoins of treatment. Radiother oncol.1988; 11:297-303 Mignote.H et Bremond.A

16. Cancers du sein opérable : notions générales sur les techniques chirurgicales. Encycl.Med.Chir (elseiver, Paris), Gynécologie $1998 ; 41: 471-81$

17. Helfre $\mathrm{S}$, Ginestet $\mathrm{C}$ et Carrie $\mathrm{C}$ Techniques d'irradiation du cancer du sein et de ses métastases. Encycl.Med.Chir. (Elseveir,Paris) 1996 ;871-A-30 :5p J.H.Bijek, JS.Aucouturier et al

18. Lymphocèles axillaires après curage ou prélèvement du ganglion sentinelle en cas de cancer du sein. Bull Cancer $2005 ; 92(2): 179-83$

19. Clough KB, Nos C, Fitoussi A, Couturaud B, Inguenault C, Sarfati I. Partial reconstruction after conservative treatment for breast cancer: classification of sequelae and treatment options. Ann Chir Plast Esthet. 2008;53(2):88-101 